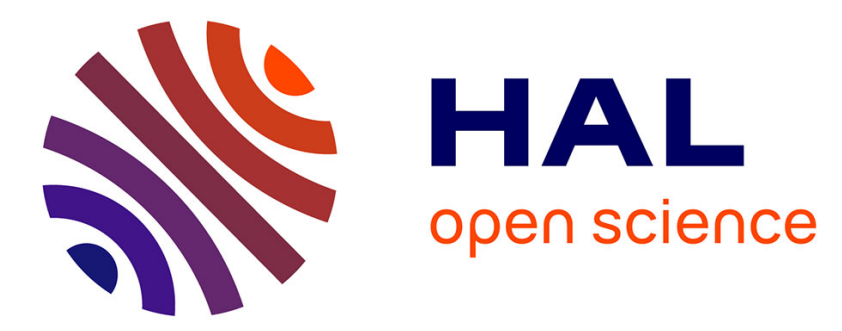

\title{
ROC-based cost-sensitive classification with a reject option
}

Clément Dubos, Simon Bernard, Sébastien Adam, Robert Sabourin

\section{To cite this version:}

Clément Dubos, Simon Bernard, Sébastien Adam, Robert Sabourin. ROC-based cost-sensitive classification with a reject option. 23rd IEEE International Conference on Pattern Recognition (ICPR), Dec 2016, Cancun, Mexico. 10.1109/ICPR.2016.7900146 . hal-02088187

\section{HAL Id: hal-02088187}

\section{https://hal-normandie-univ.archives-ouvertes.fr/hal-02088187}

Submitted on 2 Apr 2019

HAL is a multi-disciplinary open access archive for the deposit and dissemination of scientific research documents, whether they are published or not. The documents may come from teaching and research institutions in France or abroad, or from public or private research centers.
L'archive ouverte pluridisciplinaire HAL, est destinée au dépôt et à la diffusion de documents scientifiques de niveau recherche, publiés ou non, émanant des établissements d'enseignement et de recherche français ou étrangers, des laboratoires publics ou privés. 


\title{
ROC-based cost-sensitive classification with a reject option
}

\author{
Clément Dubos* ${ }^{* \dagger}$,Simon Bernard*,Sébastien Adam*,Robert Sabourin ${ }^{\dagger}$ \\ *Université de Rouen, LITIS (EA 4108), BP 12 - 76801 Saint-Étienne du Rouvray, France \\ clement.dubos@etu.univ-rouen.fr, simon.bernard@univ-rouen.fr, sebastien.adam@univ-rouen.fr \\ ${ }^{\dagger}$ Laboratoire d'Imagerie, de Vision et d'Intelligence Artificielle, \\ École de Technologie Supérieure, Université du Québec, Montreal, Canada \\ robert.sabourin@etsmtl.ca
}

\begin{abstract}
In many real-world classification tasks, such as medical diagnosis, it is crucial to take into account misclassification costs for designing an accurate classification system. Nevertheless, begin able to reject a sample is also often needed in order to avoid a very risky prediction error. In that case, a cost-sensitive classifier must embed a rejection mechanism, that takes into account the rejection costs as well as the misclassification costs. In binary classification, the ROC space has shown to be very powerful for designing cost-sensitive classifiers, but it has been poorly exploited for designing classifiers able to reject. The purpose of this work is to extend a ROC-based ensemble method recently proposed, called the ROC Front method, with a cost-sensitive rejection mechanism. This approach compares favorably to the state-ofthe-art ROC-based rejection rule recently proposed for binary cost-sensitive classification. It is also more robust as it allows to design an accurate classifier for all cost-sensitive situations contrary to the state-of-the-art method that fails in many cases, as for example with small datasets.
\end{abstract}

\section{INTRODUCTION}

Many real-world classification problems naturally exhibit imbalanced misclassification costs. Medical diagnosis is a typical example for which predicting a healthy condition for a patient who actually suffers from a serious pathology is obviously more dangerous than the opposite situation. For such cases, a wide variety of classifiers exist that take into account some predefined costs, associated to each of the possible classification errors. However, even by focusing on specific types of error, at the expense of the others, it may be difficult to completely avoid very costly prediction errors. In the previous example of medical diagnosis, one single diagnosis error can imply very serious consequences. In such a situation, it is desirable to be able not to predict any of the healthy/pathological classes, instead of taking the risk to predict 'healthy' instead of 'pathological'. Consequently, in addition to cost-sensitivity, classifiers must be able to reject a sample when the risk of being wrong is critical.

In the cost-sensitive binary classification framework, the Receiver Operating Characteristic (ROC) space has shown to be very powerful for dealing with imbalanced misclassification costs ([1]). The reason is that it allows to describe the performance of binary classifiers at different operating points, i.e. considering different cost ratios. However, it has been poorly exploited for incorporating a reject option in the costsensitive framework. The main reason is that the ROC space does not naturally allows to consider the reject option as a third possible outcome of binary classifiers.

Consider a binary classification task, where a classifier predicts either the positive $(P)$ or the negative $(N)$ class. In a cost-sensitive framework, the misclassification costs are defined in a matrix, as shown in Table Ia. When adding the ability to reject, a third column has to be defined for the cost of rejecting samples from each of the two classes, as shown in Table Ib. The traditional 2D ROC space allows the

TABLE I: Cost matrices definition. $C_{F N}$ denotes the cost of predicting negative instead of positive, $C_{F P}$ the cost of predicting positive instead of negative, $C_{T P}$ and $C_{T N}$ are the profits associated to correct predictions, and $C_{R P}$ (resp. $C_{R N}$ ) denotes the cost of rejecting a positive (resp. negative) sample. (a) Traditional Cost Matrix

\begin{tabular}{|c||c|c|}
\hline & $\hat{P}$ & $\hat{N}$ \\
\hline \hline$P$ & $C_{T P}$ & $C_{F N}$ \\
\hline$N$ & $C_{F P}$ & $C_{T N}$ \\
\hline
\end{tabular}

(b) Cost Matrix with a reject option

\begin{tabular}{|c||c|c|c|}
\hline & $\hat{P}$ & $\hat{N}$ & $\hat{R}$ \\
\hline \hline$P$ & $C_{T P}$ & $C_{F N}$ & $C_{R P}$ \\
\hline$N$ & $C_{F P}$ & $C_{T N}$ & $C_{R N}$ \\
\hline
\end{tabular}

representation of a classifier ability to handle $C_{F P}$ and $C_{F N}$. Nevertheless, if one wants to transpose the ROC space in the Table $\mathrm{Ib}$ case, he will have to add 2 more dimensions, corresponding to $C_{R P}$ and $C_{R N}$.

As far as we know, very few works have proposed a rejectbased extension of the ROC space ([2], [3]). In both [2] and [3], a third dimension, related to the rejection performance, is added to the regular 2D ROC space. In the first work ([2]), this third dimension represents the ability of an operating point to reject a sample predicted with a low confidence. However, it does not allow to take into account cost matrices like the one in Table Ib, since two more dimensions are needed to separately consider $C_{R P}$ and $C_{R N}$, instead of just one.

In the second work ([3]), the third dimension represents the rates of samples predicted as positive whereas they are expected to be rejected. This extended ROC space is used for outlier detection, where samples are classified either as positive or negative whereas they may actually belong to a third unseen class. This case would correspond to adding a new row in the Table Ia, instead of a new column as it is the case in our context. 
Tortorella proposes in [4] another type of ROC-based rejection mechanism. The traditional 2D ROC space is used to design a reject rule that leans on two decision thresholds applied on the binary classifier outcomes. It has been shown in [5] that this method is theoretically equivalent to the optimal Chow's reject rule proposed in [6], for binary classification. However this method is ineffective in two types of situation: (i) when the Equation 8 is not true (cf. Section II) and (ii) when the dataset used for the ROC analysis is too small to correctly estimate the ROC curve. In that case, the two decision thresholds are most of the times equals and irrelevant.

In a recent paper ([7]), we have shown that, for costsensitive classification, it can be more efficient to exploit the ROC space for learning a pool of classifiers, instead of only focusing on the decision thresholds proposed in the ROC curve of a single classifier. The purpose of the present work is to extend the method in [7] with a rejection mechanism that is able to jointly take into account the misclassification and rejection costs as defined in the Table Ib. The proposed method shows a significant improvement over the Tortorella's method, and allows to give an accurate cost-sensitive solution in all cases, in particular when the dataset is small.

The rest of the paper is organized as follows: the next section explains the Tortorella's method for designing a reject rule thanks to a ROC analysis; Section III presents the proposed approach; and Section IV details the experimental comparison between both methods, i.e. Tortorella's reject rule and our approach.

\section{ROC-BASED REJECT RULE (TORTORELLA'S METHOD)}

A natural way to make any binary classifier cost-sensitive is to optimize a decision threshold on its outcome, according to a given cost matrix. This allows to train the classifier regardless the costs, and to find the decision threshold that best suits to the misclassification costs afterwards. To do so, the ROC space is often used, since it allows to represent any binary classification system via its ability to recognize both classes: the first class through the True Positive Rate (TPR) and the second class through the False Positive Rate (FPR). Such a classification system, that gives a prediction $\hat{y}$ for any instance $\mathbf{x}$, will be represented in the ROC space by a $2 \mathrm{D}$ point, as illustrated in Figure 1.

Consider now that a classifier gives an output score $h(\mathbf{x})$ on any instance $\mathbf{x}$. For having a prediction $\hat{y}$, a threshold $t$ has to be set on $h(\mathbf{x})$, such that $\hat{y}=P$ if $h(\mathbf{x})>t, \hat{y}=N$ else. A typical way to represent the performance of such a classifier in the ROC space is to consider all the possible thresholds, and to draw the corresponding ROC curve, as illustrated on Figure 2. In that way, it is easy to find the operating point of this curve (and thus the corresponding threshold $t$ ) that best suits to given misclassification costs. This is usually achieved using iso-performance lines ([8]) defined by a slope given by

$$
m=\frac{p(N) C_{F P}}{p(P) C_{F N}}
$$

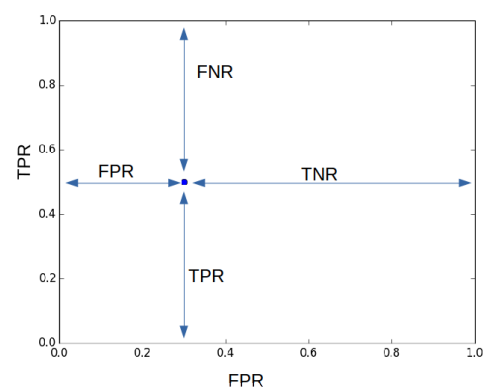

Fig. 1: Representation of a classifier system in the ROC space. $F P R, T P R, F N R$ and $T N R$ respectively denotes the false positive, true positive, false negative and true negatives rates.

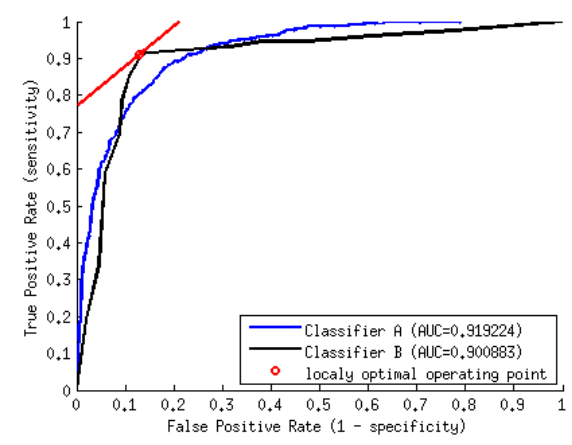

Fig. 2: ROC curves and iso-performance lines

where, $p(P)$ and $p(N)$ are the prior probabilities of the positive and the negative classes. The best threshold is given by the operating point for which the iso-performance line is tangent to the ROC curve, as illustrated in Figure 2.

The idea behind the method proposed in [4], denoted $R B R$ hereafter, is to extend the iso-performance line principle for finding two decision thresholds, $t_{1}$ and $t_{2}$, in such a way that the final prediction $\hat{y}$ is defined by:

$$
\hat{y}= \begin{cases}P & \text { if } h(\mathbf{x})>t_{1} \\ R & \text { if } t_{2}<h(\mathbf{x})<t_{1} \\ N & \text { else }\end{cases}
$$

These thresholds $t_{1}$ and $t_{2}$ can be find using two isoperformance lines, the slopes of which are:

$$
m_{1}=-\frac{p(N) C_{T N}^{\prime}}{p(P) C_{F N}^{\prime}} \quad \text { and } \quad m_{2}=-\frac{p(N) C_{F P}^{\prime}}{p(P) C_{T P}^{\prime}}
$$

where $C_{T N}^{\prime}, C_{F N}^{\prime}, C_{F P}^{\prime}$ and $C_{T P}^{\prime}$ are modified costs, as:

$$
\begin{aligned}
& C_{T N}^{\prime}=C_{T N}-C_{R N} \\
& C_{F P}^{\prime}=C_{F P}-C_{R N} \\
& C_{F N}^{\prime}=C_{F N}-C_{R P} \\
& C_{T P}^{\prime}=C_{T P}-C_{R P}
\end{aligned}
$$

The threshold $t_{1}$ (resp. $t_{2}$ ) is found with the $m_{1}$ (resp. $m_{2}$ ) iso-performance line. 
Note that there are cases for which it is not possible to find two consistent thresholds $t_{1}$ and $t_{2}$. When determining both values, three different situations can be encountered:

1) $t_{1}<t_{2}$

2) $t_{1}=t_{2}$

3) $t_{1}>t_{2}$

In the second and third situations, the prediction function of Equation 2 can not be applied. In that case, Tortorella suggests in [4] not to perform any rejection, and to use instead the regular method with only one threshold. Two types of situations can led to these situations: (i) when Equation 8 is not true and (ii) when the dataset is too small. For further explanations, please see [4].

$$
\frac{C_{T N}^{\prime}}{C_{F N}^{\prime}}>\frac{C_{F P}^{\prime}}{C_{T P}^{\prime}}
$$

\section{REJeCting With ROC-BASED COST-SENSitive CLASSIFIERS}

In [9], [7], we have proposed an ensemble method for costsensitive classification, called the ROC Front method. The rationale behind this method is to replace the traditional decision parameter optimization by a model selection approach, based on an ensemble of cost-sensitive classifiers optimized in the ROC space. This approach has shown to significantly improve the cost-sensitive results over the decision parameter optimization approach. The goal of the method proposed in this paper is to extend this ROC-based model selection with a rejection mechanism.

What is called ROC Front is an ensemble of diverse costsensitive classifiers, that proposes a variety of solutions that suit to different cost-sensitive scenarios. When a cost matrix is considered, the most suitable classifier is selected accordingly in this ensemble. For adding a reject option, a second stage is added, composed with the following steps:

1) All pairs of classifiers from the ROC front are generated. Each pair of classifiers $H_{i j}=\left(h_{i}, h_{j}\right)$ is used as a combining classifier such that the prediction is obtained following the decision function:

$$
\hat{y}= \begin{cases}P & \text { if } h_{i}(\mathbf{x})=P \text { and } h_{j}(\mathbf{x})=P \\ N & \text { if } h_{i}(\mathbf{x})=N \text { and } h_{j}(\mathbf{x})=N \\ R & \text { else }\end{cases}
$$

2) These $H_{i j}$ classifiers are projected in a 4D ROC space, where the dimensions correspond to the TPR, FPR, $R P R$ and $R N R$, where $R P R$ and $R N R$ stand for the rejected positive rates and rejected negative rates. In that extended ROC Space, the point $P_{o}$ of coordinates $(1,0,0,0)$ corresponds to a classifier that always predicts the correct class for any new instance, and that never reject. Any other points of this space is a particular cost-sensitive classifier, represented by its 4 rates ( $T P R$, $F P R, R P R, R N R$ ). The closer to $P_{o}$, the more accurate this classifier will be. However, as for the ROC Front method, the goal here is to obtain a diverse pool of classifiers, well spread all across the 4D ROC space.

\begin{tabular}{|l|c|c|c|c|}
\hline Dataset & Instances & Positive & Negative & features \\
\hline Pima & 768 & 268 & 500 & 8 \\
\hline Heart & 270 & 120 & 150 & 13 \\
\hline Australian & 690 & 383 & 307 & 14 \\
\hline Diabetes & 768 & 268 & 500 & 8 \\
\hline a1a & 1605 & 395 & 1210 & 123 \\
\hline a2a & 2265 & 572 & 1693 & 123 \\
\hline Sonar & 208 & 97 & 111 & 60 \\
\hline Splice & 1000 & 517 & 483 & 60 \\
\hline Seismic-bumps & 2584 & 2414 & 170 & 19 \\
\hline Spectf & 349 & 254 & 95 & 44 \\
\hline Spambase & 4601 & 1813 & 2788 & 57 \\
\hline German & 1000 & 300 & 700 & 24 \\
\hline
\end{tabular}

TABLE II: Datasets

3) Among all the $H_{i j}$ classifiers, a new 4D ROC Front is built with the non-dominated solutions, in the sense of Pareto-domination as defined in the multiobjective optimization literature (please see [10] for a formal definition of Pareto-domination). In practice, this means that the $H_{i j}$ classifiers that can never be considered to be the best solution for at least one cost-sensitive scenario, are discarded from the 4D ROC Front.

This ROC Front variant, with reject option, is denoted $R F R$ in the following.

Finally, when a given cost matrix is considered, the model selection process is applied in the same way it is done in [9], [7], that is to say by minimizing the loss function defined by:

$\mathcal{L}\left(H_{i j}, D\right)=p(P)\left(\sum_{j=1 . .3} C_{1 j} R_{1 j}\right)+p(N)\left(\sum_{j=1 . .3} C_{2 j} R_{2 j}\right)$

where $D$ is a dataset, $C_{1 j}$ (resp. $C_{2 j}$ ) corresponds to the costs in the first (resp. second) row of the Table Ib matrix, and $R_{1 j}$ (resp. $R_{2 j}$ ) are the corresponding rates, estimated on $D$. For example, $C_{11}$ is $C_{T P}$ in Table $\mathrm{Ib}$, and $R_{11}$ is the true positive rate of $H_{i j}$, estimated on $D$.

\section{EXPERIMENTS}

\section{A. Experimental protocol}

For evaluating both the $R B R$ and $R F R$ methods, SVM classifiers with a Radial Basis Function (RBF) Kernel have been used as base classifiers. The reason of this choice is that these classifiers are naturally cost-sensitive through their 3 hyperparameters: $\gamma, C^{+}$and $C^{-}$(please see [7] for additional explanations on these hyperparameters). By tuning these values, in particular $C^{+}$and $C^{-}$, one can make such an SVM classifier suits to given misclassification costs. As a consequence, the ROC front is made up with an ensemble of SVM classifiers, all trained with different triplets of hyperparameters $\left(\gamma, C^{+}, C^{-}\right)$. In the whole experiments, all the SVM has been implemented via the LibSVM software ([11]).

As for the experimental protocol, it has been inspired by the protocol used in [4]. First, 12 datasets have been selected from the UCI repository ([12]), and are described in Table II. For both methods, an independent validation set is required for finding the $t_{1}$ and $t_{2}$ in $R B R$, and for evaluating and selecting 


\begin{tabular}{|l||c|c|c|c|c|}
\hline & $C T P / N$ & $C F N$ & $C R P$ & $C F P$ & $C R N$ \\
\hline \hline CM1 & $\mathrm{U}(-10,0)$ & $\mathrm{U}(0,50)$ & 1 & $\mathrm{U}(0,50)$ & 1 \\
\hline CM2 & $\mathrm{U}(-10,0)$ & $\mathrm{U}(0,50)$ & 1 & $\mathrm{U}(0,100)$ & 1 \\
\hline CM3 & $\mathrm{U}(-10,0)$ & $\mathrm{U}(0,100)$ & 1 & $\mathrm{U}(0,50)$ & 1 \\
\hline CM4 & $\mathrm{U}(-10,0)$ & $\mathrm{U}(0,50)$ & $\mathrm{U}(0,30)$ & $\mathrm{U}(0,50)$ & $\mathrm{U}(0,30)$ \\
\hline CM5 & $\mathrm{U}(-10,0)$ & $\mathrm{U}(50,100)$ & $\mathrm{U}(0,50)$ & $\mathrm{U}(50,100)$ & $\mathrm{U}(0,50)$ \\
\hline CM6 & $\mathrm{U}(-10,0)$ & $\mathrm{U}(50,100)$ & $\mathrm{U}(0,50)$ & $\mathrm{U}(25,75)$ & $\mathrm{U}(0,50)$ \\
\hline CM7 & $\mathrm{U}(-10,0)$ & $\mathrm{U}(25,75)$ & $\mathrm{U}(0,50)$ & $\mathrm{U}(50,100)$ & $\mathrm{U}(0,50)$ \\
\hline CM8 & $\mathrm{U}(-10,0)$ & $\mathrm{U}(25,75)$ & $\mathrm{U}(0,50)$ & $\mathrm{U}(25,75)$ & $\mathrm{U}(0,50)$ \\
\hline
\end{tabular}

TABLE III: Cost Models. U denotes a uniform random selection in the corresponding interval of values. CM1 to CM4 are excerpt from [4]. In these first $4 \mathrm{CM}, C_{R P}$ is always equal to $C_{R N}$, while in CM5 to CM8, different values of $C_{R P}$ and $C_{R N}$ can be obtained.

the pairs of classifiers in $R F R$. For that reason, each datasets have been divided into 3 distinct subsets, with $60 \%$ of the instances in the training set $D_{r}, 20 \%$ in the validation set $D_{v}$, and the remaining $20 \%$ in the test set $D_{s}$. For reliability concerns, 10 random partitions of each dataset have been considered in the whole experiments.

Second, several cost matrices have been generated. For that purpose, eight types of cost matrices, called cost models (CM), have been considered. Each of these CM represents a typical imbalanced costs scenario. The first four CM are excerpt from the experimental protocol proposed in [4]. The last four CM are additional $\mathrm{CM}$ we propose to extend the analysis: none of the CM proposed in [4] correspond to situations where the reject costs are potentially imbalanced. However, it is an important feature in our context, since it may be important to focus on the rejection of one class of instances, rather than the other. Thus, it is desirable not to reject too much instances from the healthy class. As a consequence, the four additional $\mathrm{CM}$ propose situations where $C_{R P}$ and $C_{R N}$ are potentially imbalanced. In addition to this, these $\mathrm{CM}$ present a wider variety of imbalanced costs situations, with (i) both misclassification costs always higher than reject costs (CM5), (ii) one misclassification always higher than reject costs (CM6 and CM7) and (iii) potentially balanced misclassification costs (CM8). Table III summarizes the definition of these 8 cost models.

Each of these CM have been used to generate 1000 random cost matrices, leading to a total of $8 \times 1000=8000$ cost matrices. For each of them, results are averaged over the 10 random partitions described above. Consequently, both methods have been evaluated over $10 \times 8000=80000$ runs for each dataset.

Concerning the $R B R$ method, a single SVM classifier has been trained on $D_{r}$, for which the hyperparameters have been chosen according to a classical grid search procedure, as proposed in the LibSVM ([11]). Then, the thresholds $t_{1}$ and $t_{2}$ have been selected with the validation set $D_{v}$. And finally, the resulting classifier has been evaluated on $D_{s}$, by computing the cost-sensitive loss defined in Equation 10.

As for the RFR method, the ROC Front has been built on $D_{r}$, as explained in [9], [7]. The pool of classifier combinations, made up with the pairs of classifiers from this ROC front, is then evaluated on $D_{v}$, through a 5-fold cross-validation (CV) procedure. As a result, each combination is represented in the extended $4 \mathrm{D}$ ROC space by a $(T P R, F P R, R P R, R N R)$ point, each of these coordinates being averaged over the 5 folds of the $\mathrm{CV}$ procedure. Details are given by the Algorithm 1.

This algorithm takes as input a ROC Front, represented by an ensemble of hyperparameter vectors $\left(\gamma, C_{+}, C_{-}\right)$, each of which corresponding to one particular SVM classifier. Firstly, the 5 -fold cross-validation is prepared, by recording the predictions of all the classifiers from the ROC Front, on all the 5 folds (line 1 to 7 ). Secondly, all the possible pairs of classifiers from the ROC Front are generated (line 8). Thirdly, for all these pairs of classifiers, new predictions are given for each folds of the $\mathrm{CV}$ procedure, applying the decision rule given in Equation 9 (line 9 to 23). These predictions are used to build a mean confusion matrix, averaged over the rates obtained on the 5 folds. The projection of any combining classifier of the $R F R$ method in the extended 4D ROC space, is excerpt from this mean confusion matrix. Finally, the algorithm outputs the "best" combining classifiers in this ROC space, in the sense of Pareto-domination, as explain in Section III (line 24).

When the cost matrix is then considered, the most suitable pairs is selected by minimizing the loss function of Equation 10 , also estimated on $D_{v}$. Finally, the resulting combining classifier has been evaluated on $D_{s}$, as for the $R B R$ method.

\section{B. Results}

In order to assess the significance of the results, the Wilcoxon signed ranks test has been applied on each cost matrix evaluation, as in [4] and as recommended in [13] for comparison of two classifiers. Consequently, each CM, applied on each dataset, have led to 1000 significant win/loss results, obtained over the 10 random partitions. These results are gathered in Table IV. Each cell of Table IV contains 3 values: from the top to the bottom, the significant win counts for the $R F R$ method, the significant loss counts and the number of cost matrices for which both methods do not give loss results significantly different. For exhaustiveness, we also report in the last column, the total counts of win/loss/tie counts, over the 8000 matrices.

The first observation made from this table is that $R F R$ and $R B R$ do not give statistically different results in a majority of cases, i.e. $80.9 \%$ (71216 of the 88000 comparisons). Then, $R F R$ significantly outperforms $R B R$ for $17.78 \%$ of the cases (15642 over 88000 ), while $R B R$ rarely outperforms $R F R$, i.e. for $1.3 \%$ of the cases only (1142 over 88000 ). To sum it up, the proposed approach is at least as accurate as the Tortorella's approach, and gives a significant improvement for more than 1 out of 6 cases, and so for a wide variety of imbalanced costs.

A strong advantage of the $R F R$ method is that it never fails to give a suitable cost-sensitive solutions, whatever the priors and the costs. On the contrary, as explained at the end of Section II, it is sometimes impossible for the $R B R$ approach 


\begin{tabular}{|l|c|c|c|c|c|c|c|c|c|}
\hline Files & CM1 & CM2 & CM3 & CM4 & CM5 & CM6 & CM7 & CM8 & Total \\
\hline \multirow{3}{*}{ Heart } & 36 & 26 & 26 & 333 & 0 & 0 & 227 & 260 & 908 \\
& 11 & 8 & 68 & 1 & 0 & 1 & 0 & 0 & 89 \\
& 953 & 966 & 906 & 666 & 1000 & 999 & 773 & 740 & 7003 \\
\hline \multirow{3}{*}{ Australian } & 84 & 51 & 53 & 418 & 1 & 26 & 253 & 262 & 1148 \\
& 0 & 0 & 0 & 0 & 0 & 0 & 0 & 0 & 0 \\
& 916 & 949 & 947 & 582 & 999 & 974 & 747 & 738 & 6852 \\
\hline \multirow{3}{*}{ Diabetes } & 98 & 53 & 63 & 473 & 0 & 104 & 255 & 262 & 1308 \\
& 4 & 5 & 6 & 1 & 6 & 1 & 2 & 1 & 26 \\
& 898 & 942 & 931 & 526 & 994 & 895 & 743 & 737 & 6666 \\
\hline \multirow{3}{*}{ German } & 86 & 61 & 48 & 399 & 0 & 6 & 258 & 263 & 1121 \\
& 1 & 0 & 2 & 0 & 5 & 0 & 0 & 0 & 8 \\
& 913 & 939 & 950 & 601 & 995 & 994 & 742 & 737 & 6871 \\
\hline \multirow{3}{*}{ Pima } & 0 & 0 & 0 & 0 & 0 & 0 & 0 & 0 & 0 \\
& 0 & 0 & 0 & 0 & 0 & 0 & 0 & 0 & 0 \\
& 1000 & 1000 & 1000 & 1000 & 1000 & 1000 & 1000 & 1000 & 8000 \\
\hline \multirow{3}{*}{ Sonar } & 31 & 20 & 28 & 327 & 0 & 1 & 221 & 253 & 881 \\
& 6 & 11 & 5 & 4 & 7 & 0 & 12 & 14 & 59 \\
& 963 & 969 & 967 & 669 & 993 & 999 & 767 & 733 & 7060 \\
\hline \multirow{3}{*}{ Spectf } & 394 & 184 & 558 & 867 & 628 & 850 & 575 & 930 & 4986 \\
& 0 & 0 & 0 & 0 & 0 & 0 & 0 & 0 & 0 \\
& 606 & 816 & 442 & 133 & 372 & 150 & 425 & 70 & 3014 \\
\hline \multirow{3}{*}{ Spambase } & 126 & 130 & 81 & 402 & 7 & 7 & 263 & 263 & 1279 \\
& 0 & 0 & 1 & 0 & 0 & 0 & 0 & 0 & 1 \\
& 874 & 870 & 918 & 598 & 993 & 993 & 737 & 737 & 6720 \\
\hline \multirow{3}{*}{ Splice } & 65 & 51 & 45 & 361 & 0 & 0 & 254 & 261 & 1037 \\
& 209 & 262 & 212 & 36 & 68 & 72 & 23 & 11 & 893 \\
& 726 & 687 & 743 & 603 & 932 & 928 & 723 & 728 & 6070 \\
\hline \multirow{3}{*}{ A1a } & 120 & 88 & 68 & 549 & 8 & 206 & 308 & 280 & 1627 \\
& 0 & 0 & 0 & 0 & 0 & 0 & 0 & 0 & 0 \\
& 880 & 912 & 932 & 451 & 992 & 794 & 692 & 720 & 6373 \\
\hline \multirow{3}{*}{ A2a } & 112 & 67 & 63 & 476 & 0 & 88 & 279 & 262 & 1347 \\
& 4 & 5 & 2 & 5 & 10 & 6 & 1 & 33 & 66 \\
& 884 & 928 & 935 & 519 & 990 & 906 & 720 & 705 & 6587 \\
\hline
\end{tabular}

TABLE IV: Results of the comparison as win/loss/tie counts over the $8 \times 11=88000$ cost matrices

\begin{tabular}{|l|c|c|c|c|c|c|c|c|}
\hline Files & CM1 & CM2 & CM3 & CM4 & CM5 & CM6 & CM7 & CM8 \\
\hline Heart & 22 & 16 & 22 & 25 & 0 & 0 & 0 & 0 \\
\hline Australian & 58 & 23 & 38 & 108 & 0 & 25 & 0 & 0 \\
\hline Diabetes & 75 & 30 & 52 & 163 & 0 & 104 & 1 & 0 \\
\hline German & 52 & 22 & 31 & 86 & 0 & 5 & 5 & 1 \\
\hline Pima & 0 & 0 & 0 & 0 & 0 & 0 & 0 & 0 \\
\hline Sonar & 15 & 10 & 23 & 22 & 5 & 1 & 13 & 13 \\
\hline Spectf & 240 & 114 & 155 & 541 & 577 & 776 & 323 & 653 \\
\hline Spambase & 63 & 41 & 37 & 84 & 0 & 7 & 0 & 0 \\
\hline Splice & 50 & 32 & 35 & 55 & 3 & 11 & 0 & 0 \\
\hline A1a & 96 & 52 & 56 & 238 & 8 & 206 & 54 & 18 \\
\hline A2a & 89 & 45 & 53 & 171 & 8 & 94 & 26 & 33 \\
\hline
\end{tabular}

TABLE V: Counts of cost matrices for which a 0-reject classifier has been used in replacement of the $R B R$ method

to find consistent thresholds such that $t_{1}<t_{2}$. Every time such a situation has been encountered in our experiments, a 0 -reject classifier has been used instead, with only one decision threshold $t$ used, and found by minimizing the loss function. The number of cases for which the $R F R$ method is compared to the 0-reject classifier in replacement of the $R B R$ method are summarized in Table V. These counts confirm the conclusion of Tortorella in [4]: it is more likely with the cost model CM4 than with the cost models CM1 to CM3, that the condition for finding the thresholds $t_{1}$ and $t_{2}$, expressed by Equation 8, are not met.

Another interesting observation made from this table is that $R B R$ often fails to find a consistent pair of threshold $\left(t_{1}, t_{2}\right)$ for the Spectf dataset. From our point of view, it highlights another drawback of the method: when there is only few validation instances available, which is the case of the Spectf dataset due to the low number of negative instances, the ROC curve does not contain a sufficient number of operating points to propose enough different cost-sensitive solutions. This is well illustrated in Figure 3, which represents the ROC curve of the classifier used in the $R B R$ method for the Spectf dataset (the blue points). The potentially selected operating points for any cost-sensitive scenarios are the points lying on the red curve, called the convex hull. One can see that only 4 points are available here. In that case, it is likely that the same operating point will be selected for both thresholds. This pathological case is also discussed in [4]. On the opposite, the corresponding ROC Front used in $R F R$, made up with several cost-sensitive classifiers (the red points), proposes a lot more diverse ensemble of solutions. The reason is that the number of 


\section{Algorithm 1 ROC Front with reject option $(R F R)$}

Require: $D_{v}$ : a Dataset

Require: $F$ : the number of folds for the cross-validation $(\mathrm{CV})$ procedure

Require: $\Theta=\left\{\theta_{i}, i=1 . . K\right\}$ : a pool of $\mathrm{K}$ triplets of hyperparameters, composing the ROC Front, where $\theta_{i}=\left(C_{i}^{+}, C_{i}^{-}, \gamma_{i}\right)$

Data $\Omega=\left\{\left(\theta_{i}, \theta_{j}\right), i, j=1 . . K, i \geq j\right\}$ : a pool of $K(K+1) / 2$ pairs of classifiers from $\Theta$

Data $T_{n}$ : the $n^{t h}$ fold of $\mathrm{CV}$ procedure applied on $D_{v}$, with $n=$ $1 . . F$

Data $P_{n, i, j}$ : the prediction of the $i^{t h}$ classifier, on the $j^{\text {th }}$ instance of the $n^{\text {th }}$ fold

Data $\operatorname{Conf}_{n, i}$ : the confusion matrix of the $i^{\text {th }}$ classifier, obtained on the $n^{t h}$ fold

Function Partition For $C V\left(D_{v}, F\right)$ : outputs $F$ distincts folds following a classical $\mathrm{CV}$ procedure.

Function $\operatorname{Train}(\theta, T)$ : outputs a classifier trained on the dataset $T$, with the $\theta$ hyperparameter values.

Function Predict $(\theta, T)$ : outputs the prediction of a given SVM classifier $\theta$ for all the instances in $T$

Function bestCombiningClassif $($ Conf,$\Omega)$ : outputs the subset of $\Omega$ that represent the best solutions in the multiobjective optimization sense (Pareto optimality).

Ensure: $\Gamma=\left\{\left(\theta_{i}, \theta_{j}\right), \theta_{i}, \theta_{j} \in \Omega\right\}$ : the ensemble of pairs of SVM classifiers, composing the $R F R$ ensemble.

1: $T_{n=1 . . F} \leftarrow$ PartitionFor $C V\left(D_{v}, F\right)$

2: for $\mathrm{i}$ from 1 to $\mathrm{K}$ do

3: $\quad$ for $\mathrm{n}$ from 1 to $\mathrm{F}$ do

4: $\quad \vartheta \leftarrow \operatorname{Train}\left(\theta_{i}, \cup_{s=1, s \neq n}^{F} T_{s}\right)$

5: $\quad P_{n, i, .} \leftarrow \operatorname{Predict}\left(\vartheta, T_{n}\right)$

6: end for

7: end for

8: $\Omega \leftarrow\left\{\left(\theta_{i}, \theta_{j}\right), \theta_{i}, \theta_{j} \in \Theta, i, j=1 . . K, i \geq j\right\}$

9: for $i=1 . . K$ do

10: for $\mathrm{j}=\mathrm{i} . . \mathrm{K}$ do

11: $\quad$ for $\mathrm{n}$ from 1 to $\mathrm{F}$ do

12: $\quad$ for $\mathrm{p}$ from 1 to $\left|T_{n}\right|$ do

13: $\quad$ if $P_{n, i, p} \neq P_{n, j, p}$ then

14: $\quad$ Preject $_{p} \leftarrow$ Rejection

15: $\quad$ else

$16:$

17:

18:

19:

20:

21:

22:

23: end for

24: $\Gamma \leftarrow$ bestCombiningClassif $(\overline{\text { Conf. }}, \Omega)$

these classifiers does not depend on the number of validation instances available. This explains the particularly good results of $R F R$ on the Spectf dataset, as shown in Table IV.

\section{CONCLUSION}

In this paper, a ROC-based ensemble method has been proposed for cost-sensitive classification with rejection. This method relies on a pool of cost-sensitive classifiers, called the ROC Front. Pairs of classifiers from the ROC Front are combined to add the ability to reject. The combining classifiers that proposes the best solution for tackling particular cost-sensitive situations, i.e. with given (imbalanced) misclassification and

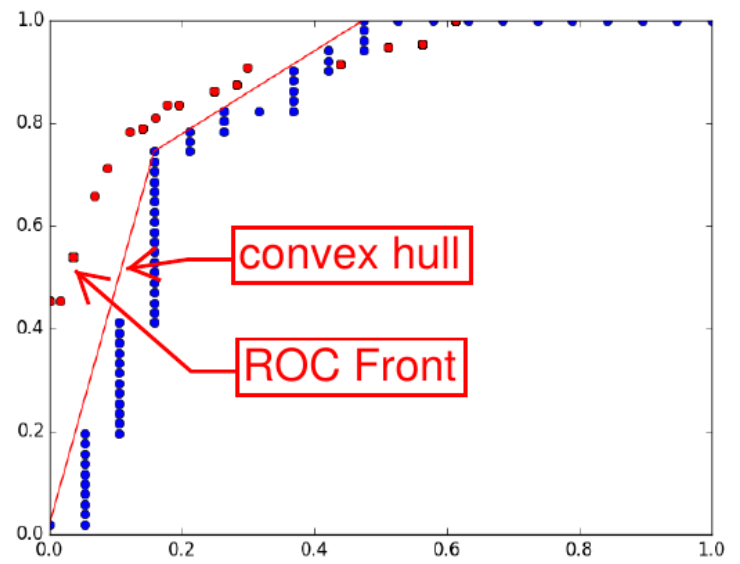

Fig. 3: Illustration of the pathological case for $R B R$, where the ROC curve does not contain enough operating points for selecting two differents thresholds

rejection costs, are retained to compose a new ROC Front with reject option.

The proposed approach compares favorably to the state-ofthe-art ROC-based reject rule, with two noticeable improvements: (i) it gives a significant improvement in terms of costsensitive performance and (ii) it is always able to propose an efficient solution for any cost-sensitive situation, contrary to the state-of-the-art method that regularly fails to introduce the reject option regarding the costs ratios.

\section{REFERENCES}

[1] T. Fawcett, "An introduction to roc analysis," Pattern Recognition Letters, vol. 27, no. 8, pp. 861-874, 2006.

[2] S. Alsing, E. Blasch, and K. Bauer, "Three-dimensional receiver operating characteristic (roc) trajectory concepts for the evaluation of target recognition algorithms faced with the unknown target detection problem," pp. 449-458, 1999.

[3] T. Landgrebe, D. Tax, P. Paclk, and R. Duin, "The interaction between classification and reject performance for distance-based reject-option classifiers," Pattern Recognition Letters, vol. 27, pp. 908-917, 2006.

[4] F. Tortorella, "A roc-based reject rule for dichotomizers," Pattern Recognition Letters, vol. 26, no. 2, pp. 167-180, 2005.

[5] C. Santos-Pereira and A. Pires, "On optimal reject rules and roc curves," Pattern Recognition Letters, vol. 26, no. 7, pp. 943-952, 2005.

[6] C. Chow, "On optimum recognition error and reject tradeoff," IEEE Transactions on Information Theory, vol. 16, no. 1, pp. 41-46, 1970.

[7] S. Bernard, C. Chatelain, S. Adam, and R. Sabourin, "The multiclass roc front method for cost-sensitive classification," Pattern Recognition, vol. 46, pp. 46-60, 2016.

[8] F. Provost and T. Fawcett, "Robust classification for imprecise environments," Machine Learning, vol. 42, no. 3, pp. 203-231, 2001.

[9] C. Chatelain, S. Adam, Y. Lecourtier, L. Heutte, and T. Paquet, "A multimodel selection framework for unknown and/or evolutive misclassification cost problems," Pattern Recognition, vol. 43, no. 3, pp. 815-823, 2010.

[10] K. Deb, Multi-Objective Optimization Using Evolutionary Algorithms. New York, NY, USA: John Wiley \& Sons, Inc., 2001.

[11] C. Chang and C. Lin, "Libsvm: A library for support vector machines," ACM Transaction on Intelligent Systems and Technology, vol. 2, no. 3, pp. 1-27, 2011.

[12] K. Bache and M. Lichman, "UCI Machine Learning Repository," 2013. [Online]. Available: http://archive.ics.uci.edu/ml

[13] J. Demsar, "Statistical comparisons of classifiers over multiple data sets," Journal of Machine Learning Research, vol. 7, pp. 1-30, 2006. 